\title{
Research on the Support Model of Large Equipment Emergency Spare Parts under Fuzzy Demand
}

\author{
Jianhua Yang, Zhichao Ma, Yang Song \\ Donlinks School of Economics and Management University of Science and Technology Beijing (China)
}

yangib@ustb.edu.cn, mzco811@126.com, 416955174@qq.com

Received: January 2015

Accepted: April 2015

\section{Abstract:}

Purpose: Aim at making a support scheme for emergency spare parts when large equipment spare parts supply network faced with large-scale emergency events.

Design/methodology/approach: In order to analyze the model, we establish the spare parts security model under network supply conditions to respond emergency in case of fuzzy demand. And in end of the paper, we adopt an improved genetic algorithm to solve the problem.

Findings: Considering emergency spare parts support problem from three aspects including satisfaction of time, satisfaction of demand and emergency cost constraints, which makes decision-making process more accord with reality condition, we can get a more realistic solution for the decision makers.

Originality/value: Considering the occurrence of emergency and adopting information entropy theory to order the weight of emergency maintenance station in priority sequence, this paper presented emergency response time and demand satisfaction function, which uses the time, demand satisfaction and the cost restrictor as objective functions, we have constructed the spare parts support model under fuzzy demand to solve emergency events, having expanded the scope of solution.

Keywords: fuzzy demand, satisfaction index, personality, emergency spare parts 


\section{Introduction}

The reason for the complexity of maintenance spare parts for large equipment involves many complicated factors. Some attribute to the different types and large numbers of spare parts, others point to the intricacy of maintenance work. Spare parts management is a hot topic of the research field, and the supply of spare parts affects the exertion of the equipment support ability directly. Effective spare parts management can provide resources for equipment support in time, and ensure the stable operation of system that perform the maximum efficiently under the limited support cost. The decision maker is required to put forward the corresponding plan rapidly in an unexpected event, and spare parts' supply requires fast response, accurate and reliable quantity. It is likely to miss the best opportunity of spare parts support and cause serious consequences if the decision making speed is slower. Therefore, in the process of support emergency spare parts, the improvement of equipment spare parts support work time and demand satisfaction should be considered for the problem of emergency resource optimization.

Aiming at the characteristics of multiple emergency spot and multiple resources problem, the concept of continuous feasible plan is introduced by Dai and Da (2000) to realize the solution of multiple resource allocation emergency problems, an example and the application are given, and satisfactory results are obtained. An optimal scheduling model of emergency resource allocation is put forward from the angle of time and space by Fiedrich, Gehbauer and Rickers (2000), the model uses detailed descriptions of the available resources to calculate the resource performance for different tasks related to the response. For the problem of emergency support multi-depots to single demand depots, a model for multiple targets emergency support is established by Liu, He and Shi (2001). Which is based on the fewest number of selected depots and the shortest emergency time. Considering the multiple species allocation of emergency supplies and vehicle scheduling, a mathematical model of unmet and minimum demand for emergency supplies which is established by Özdamar, Ekinci and Küçükyazici (2004), the model describes a setting that is different than the conventional vehicle routing problem. It is readily decomposed into two multi-commodity network flow problems, the first one being linear and the second integer. The problem of hybrid fuzzy clustering algorithm being used to solve the relief phase emergency point clustering and allocation of emergency supplies is put forward by Shen (2007), this paper presents an optimization approach to the operation of emergency logistics co-distribution responding to the urgent relief demands in the rescue period. A method was presented by Du and Yi (2013) which chose relative good vehicle routing in emergency conditions, considering time requirement and cost limit both while choosing vehicle routing establishing a mathematical model of multi-objective emergency logistics vehicle routing problem. Use genetic algorithms to solve the problem. For the problem of allocation of emergency supplies in an unexpected event, considering the limited capacity of vehicle scheduling, a mathematical model of taking 
the least vehicle scheduling costs and minimization of loss as the target is established by Wang, Ma and Ruan (2013). This paper used the hierarchical thinking to reduce solution space, designed the genetic algorithm for this problem, the validity of the model is verified combining with realistic distribution scheme.

Many scholars at home and abroad who have made a lot of meaningful exploration for resource emergency support in an unexpected event, most of which support the resource allocation and transportation resources with demand certainty, and the study of the degrees of satisfaction in the field of emergency currently is more focused on distribution center location problem (Tzeng, Cheng \& Huang, 2007), but demand of spare parts for large equipment is usually uncertain after the occurrence of actual emergency. Therefore, how to deal with problems of emergency spare parts support of large equipment in an unexpected event firstly need to estimate the demand of spare parts by the fuzzy method and to solve uncertainties. Aiming at problems of emergency spare parts support of large equipment in an unexpected event, a model of emergency spare parts support of large equipment is established under fuzzy demand in this paper. Considering the occurrence of emergency, the model estimates the demand of spare parts by the fuzzy method, based on the sorting of each maintenance station security priority, explores problems of emergency spare parts support from three aspects of satisfaction of time, satisfaction of demand and emergency cost constraints, which makes decision-making process more accord with the reality, so as to help decision makers to provide better emergency solution of effective maintenance resource allocation.

\section{Model}

\subsection{Problem Description and Parameter Representation}

After the occurrence of emergency event, maintenance stations need to stock emergency spare parts, and each maintenance station is taken as a point of emergency rescue. Its emergency response processes includes: firstly, it needs to count up and analyze the demand of the existing emergency resources for each maintenance station; secondly, the weights of support priority of the urgency and importance of each maintenance station is ranked based on the statistical data; thirdly, a model of emergency spare parts support of equipment is established; finally, emergency schemes are given by solving the model, and the large spare parts warehouse distribution center provides spare parts support for these emergency rescue stations. The response flow chart is shown in Figure 1. 


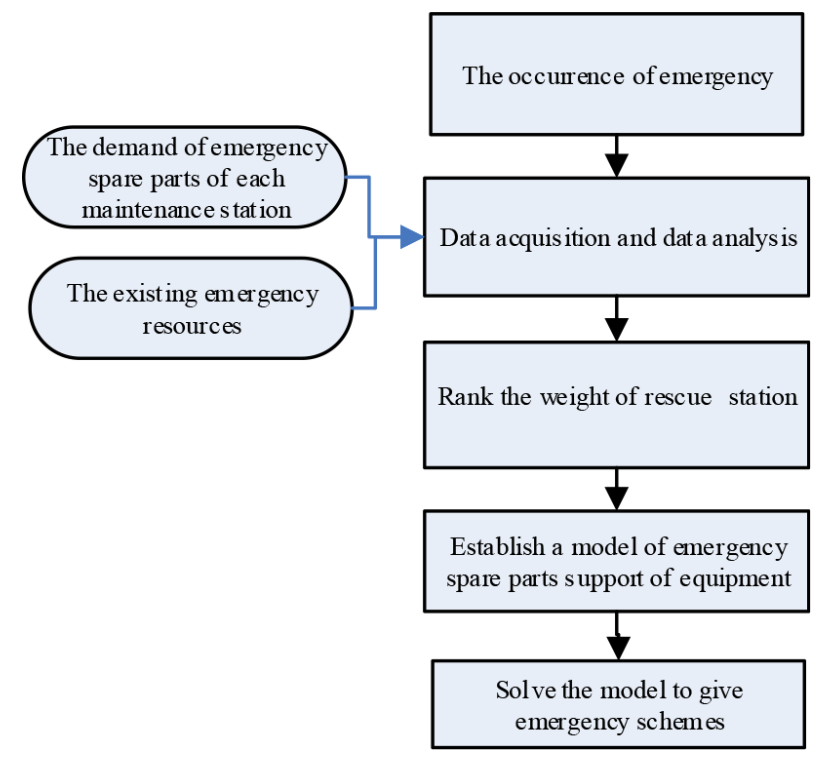

Figure 1. The flow chart of emergency response of large equipment spares

Set definition and description of model parameters:

- Emergency rescue point set: $I=\{1,2, \ldots, n\}, i \in I$.

- The spare parts warehouse distribution center set: $J=\{1,2, \ldots, m\}, j \in J$.

- $t_{i j}$ denotes generalized temporal distance from the emergency rescue station $i$ to the spare parts warehouse distribution center $j$.

- $\tilde{d}_{i}$ denotes fuzzy demand of the emergency spare parts of the emergency rescue station $i$ in the initial time.

- $x_{i j}$ denotes actual amount emergency spare parts delivery from the spare parts warehouse distribution center $j$ to the emergency rescue station $i$.

- $\quad c_{i j}$ denotes unit transportation cost from the spare parts warehouse distribution center $j$ to the emergency rescue station $i$.

- $\quad c^{*}$ denotes emergency budget made by decision-making section.

- $h_{j}$ denotes emergency spare parts storage of spare parts warehouse distribution center $j$.

- $\quad Y_{i j}$ denotes that if spare parts warehouse distribution center $j$ services the emergency rescue station $i$, so $Y_{i j}=1$, otherwise $Y_{i j}=0$.

- $W_{i}$ denotes the weights of emergency support priority of the emergency rescue station $i$.

- $R_{i}$ denotes the ratio of satisfaction and demand of the emergency rescue station $i$. 


\subsection{The Weights Rank of Support Priority of Each Maintenance Station}

When the emergency happens, spares demand of each maintenance station is different in the model of emergency spare parts support of equipment, and the distance from each maintenance station to the spare parts warehouse distribution center is also different. Maintenance personnel, transport vehicles and emergency budget are limited. So it needs to rank the weights of support priority of each maintenance station and determine the optimal allocation scheme.

Methods of determining the weight are usually applied by expert assignment method of subjectivity and analytic hierarchy process (AHP) of relative objectivity. The deficiency of expert assignment method is more optional, especially it leads to wrong evaluation results when the judgment of the experts and scholars is very different from the reality. For AHP method, due to the fluctuation of data information, imperfection and inaccuracy, the information got by further data mining which is likely to have big deviation with the real results. In order to overcome these problems, the information entropy theory based on indicators such as the spare parts demand and the existing resources etc. are applied to determine the weight, the process is as follows:

Measures belong to $k$ categories of the sample $x_{i}$ made by observations $x_{i j}$ of a sample with particular attributes are set as: $\mu_{i j}, \mu_{i j}, \ldots, \mu_{i j k}$ satisfies $0 \leq \mu_{i j k} \leq 1, \sum_{i=1}^{k} \mu_{i j k}=1$. That is, the $\left\{\mu_{i j k}\right\}$ has the nature of a certain probability.

At this point the entropy is:

$$
H=-\sum_{k=1}^{K} \mu_{i j k} \cdot \log \mu_{i j k}
$$

From the perspective of identification, various kinds of endings of randomized trials are regarded as sample classification, and any kind of measure of sample $x_{i}$ being existed in $k$ categories by attribute $I_{j}$ is $\frac{1}{k}$. Thus it can be seen that attribute $I_{j}$ has no effect on identifying the classification of sample $x_{i}$. Conversely, if one of the $\mu_{i j k}$ is one and the rest is zero, so $H=0$, which shows that attribute $I_{j}$ is important reflecting on the identification. So the value of $\mu_{i j k}$ about the $k$ th classification is more concentrated, that is the smaller the $H$ is, the more important the classification of $x$ identified by indicator $I_{j}$ is. Therefore,

$$
v_{i j}=1+\frac{1}{\log k} \sum_{k=1}^{K} \mu_{i j k} \log \mu_{i j k}
$$

Obviously $0 \leq v_{i j} \leq 1$, if the value of $v_{i j}$ is bigger, the value of $H$ is smaller, the classification of the corresponding attribute $I_{j}$ in the $x_{i}$. When $v_{i j}=1$, it shows that there is one in the $\mu_{i j}$, and 
the rest is zero. At this point, measures of sample $x$ in the $k_{1}$ th classification made by the observation of attribute $I_{j}$ is one, the rest in the other classification is zero. That is, the sample $x_{i}$ is certainly categorized into $\mathrm{K}$ categories by $I_{j}$, which indicates that $I_{j}$ has the most importance of identifying the classification of $x_{i}$. If $v_{i j}=0$, that is each $\mu_{i j}=\frac{1}{k}$, measures of the sample $x_{i}$ have been divided into all kinds of classification by $I_{j}$ as redundant attributes to remove are the same. The more concentrated the value of $\mu_{i j}$ is, the bigger function of the attribute $I_{j}$ acts on classification of $x_{i}$. Set:

$$
\mu_{i j}=v_{i j} / \sum_{j=1}^{m} v_{i j}
$$

Consequently $0 \leq \mu_{i j} \leq 1, \sum_{j=1}^{m} \mu_{i j}=1$, and the bigger value of $\mu_{i j}$ is, the more important $I_{j}$ to classification of $x_{i}$ is. For that reason the weighting vector is $\mu_{i}=\left(\mu_{i 1}, \mu_{i 2}, \ldots, \mu_{i m}\right)^{T}$.

\subsection{Time Satisfaction Degree and Demand Satisfaction Degree}

\subsubsection{Time Satisfaction Degree}

The spare parts emergency support satisfaction degree studied in this paper which is mainly time satisfaction degree and demand satisfaction degree. The customer satisfaction of emergency response time is the key factor for making decisions. In the process of handling emergency event, the distribution of the emergency spare parts may appear no symmetry information caused by the characters of emergency event. Among them, the information of timeliness is one of the key factors of emergency, and the customer satisfaction of emergency response time reflects the effect of emergency support to a certain extent. The value function is used to describe the customer satisfaction of emergency response time. The concave function of time satisfaction degree function is in incremental interval, and its convex function is in the negative incremental range. Generally, the same response time is different from different maintenance point of customer satisfaction.

The difference of emergency support objective condition leads to the value of the reference point of varying sizes in Figure 2, and the reference point of emergency maintenance point $i$ denotes the average support time from each storage center to the point $i$.

$$
T_{i a}=\sum_{j=1}^{m} \frac{t_{i j}}{m}
$$




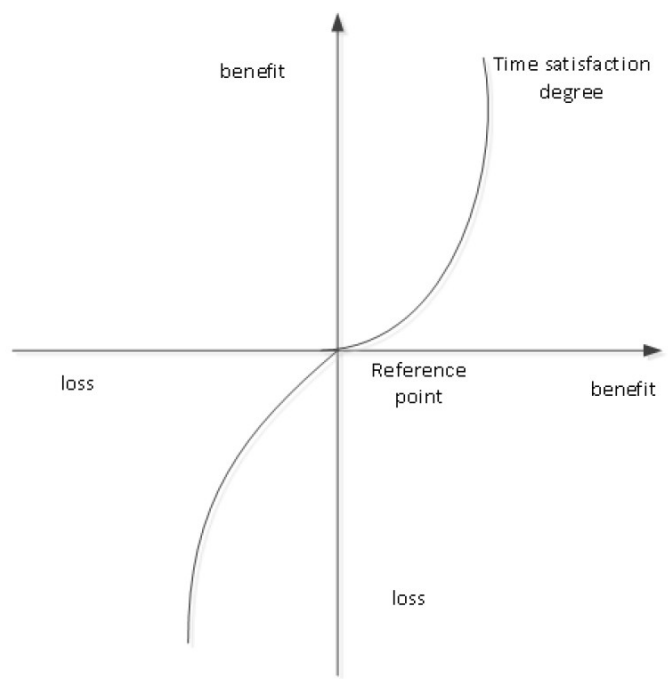

Figure 2. The emergency response time satisfaction degree function

Consequently we take point $i$ as an example, the deviation of emergency support time is defined as:

$$
T_{i}=T_{i a}-\max _{j \in J}\left\{Y_{i j} \bullet t_{i j}\right\}=\sum_{j=1}^{m} \frac{t_{i j}}{m}-\max _{j \in J}\left\{Y_{i j} \bullet t_{i j}\right\}
$$

The income function is used to measure the deviation of emergency response time for customers, which is:

$$
\mathrm{V}\left(T_{i}\right)=\left\{\begin{array}{cc}
T_{i}^{a}, & T_{i} \geq 0 \\
-\lambda\left(-T_{i}\right)^{\beta}, & T_{i}<0
\end{array}\right.
$$

\subsubsection{Demand Satisfaction Degree}

In the context of emergency spare parts support of large-scale unexpectedness case, the demand of maintenance point emergency spare parts is high and fuzzy, and the existing emergency resources are limited. So the higher spare parts demand satisfied rate is, the more the demand satisfaction degree is. The method of spare parts demand satisfaction degree in reference expresses the demand satisfaction degree by the relative demand rate function. The demand satisfaction degree function of emergency maintenance point $i$ is expressed as:

$$
\mathrm{g}(\mathrm{i})=\frac{R_{i}-\min _{i \in I}\left\{R_{i}\right\}}{\max _{i \in I}\left\{R_{i}\right\}-\min _{i \in I}\left\{R_{i}\right\}}
$$

Among them $R_{i}=\sum_{j=1}^{m} \frac{x_{i j}}{\tilde{d}_{i}}$. 


\subsection{Model Construction}

In the process of emergency support of unexpectedness case, emergency budget is also an important factor which needs to be considered. But for the timeliness of emergency spare parts support, the primary goal of the model is the least time of emergency support. Therefore, the primary goal of the model need to maximize the emergency time satisfaction degree when solving objective optimization, and then the needs of customer's satisfaction degree and the cost of emergency are considered. The mentioned model is as following:

$$
\begin{gathered}
P_{1} \quad \max \sum_{i=1}^{n} w_{i} \mathrm{~V}\left(T_{i}\right) \\
P_{2} \quad \max \sum_{i=1}^{n} w_{i} \mathrm{~g}(\mathrm{i}) \\
\text { s.t } \sum_{j=1}^{m} \sum_{i=1}^{n} x_{i j} c_{i j} t_{i j} \leq c^{*} \\
T_{i}=\sum_{j=1}^{m} \frac{t_{i j}}{m}-\max _{j \in J}\left\{Y_{i j} \bullet t_{i j}\right\} \\
\sum_{j=1}^{m} x_{i j} \leq \tilde{d}_{i}, \mathrm{i} \in I \\
\sum_{i=1}^{n} x_{i j} \leq h_{j}, \mathrm{j} \in J \\
x_{i j}\left(1-Y_{i j}\right)=0, \mathrm{i} \in I, \mathrm{j} \in J \\
Y_{i j}=0 \text { or } 1, \mathrm{i} \in I, \mathrm{j} \in J
\end{gathered}
$$

Equation (5) is time satisfaction degree objective function which express maximum customer revenue function with different weights. Equation (6) is demand satisfaction degree function which express overall maximum demand satisfaction degree of maintenance station. Equation (7) is the emergency cost of emergency budget constraint. Equation (8) is the definition of time deviation. Equation (9) denotes that the initial configuration of the emergency spare parts are in short supply of large- scale unexpectedness case. Equation (10) denotes that the amount of spare parts dispatched to various emergency maintenance point cannot exceed its own spare parts storage for each warehouse distribution center. Equation (11) denotes the consistency of values of $x_{i j}$ and $Y_{i j}$. Equation (12) denotes that $Y_{i j}$ is $0-1$ variables. 


\section{Solution of Model}

The multiple maintenance point emergency resource allocation model which is made in this paper belongs to multi-objective fuzzy constraint programming problem, which firstly needs to deal with fuzzy constraints.

\subsection{The Defuzzification of Model}

Considering the triangular fuzzy number can intuitively and scientifically denotes the characteristics of fuzzy estimated by decision-makers by the way of the most pessimistic value, the most possible value, and the most optimistic value, this paper describes the demand of emergency spare parts of unexpectedness case using the triangular fuzzy number symbolized by $\tilde{d}_{i}=\left(a_{i}, b_{i}, c_{i}\right)\left(a_{i} \leq b_{i} \leq c_{i}\right), a_{i}, b_{i}$ and $c_{i}$ respectively denotes the most pessimistic value, the most possible value, and the most optimistic value belonging to real number of emergency spare parts estimated by decision-makers of unexpectedness case), its fuzzy membership degree is:

$$
u_{d_{i}}(x)=\left\{\begin{array}{c}
x-\mathrm{a}_{i} / \mathrm{b}_{i}-\mathrm{a}_{i}, \mathrm{a}_{i} \leq x \leq \mathrm{b}_{i} \\
\mathrm{c}_{i}-x / \mathrm{c}_{i}-\mathrm{b}_{i}, \mathrm{~b}_{i} \leq x \leq \mathrm{c}_{i}
\end{array}\right.
$$

The method that was used in (Liang, 2006) mentioned in this paper is adopted to deal with fuzzy constraints. Considering comprehensively the balance between the risk preference of decision makers and possibility degree of attribute values, after confidence level $\alpha$ is given by the decision makers, $\alpha \in[0,1]$, the weighted average method is adopted to translate triangular fuzzy number to determine value. Emergency spare parts predict the demand of fuzzy:

$$
\tilde{d}_{i}=\omega_{1} \mathrm{a}_{i}+\omega_{2} \mathrm{~b}_{i}+\omega_{3} \mathrm{c}_{i}
$$

$\omega_{1}, \omega_{2}$ and $\omega_{3}$ respectively denote the weight of the most pessimistic value, the most possible value and the most optimistic value of emergency spare parts demand fuzzy number for decision makers. The most possible value, the most pessimistic value and the most optimistic value that appear in ( $\mathrm{Li} \& \mathrm{Lai}, 2000$ ) mentioned in this paper is adopted as boundary constraints of fuzzy number. The estimate of multiple fuzzy state is usually too pessimistic or optimistic, so a small weight is assigned to it. The most possible value is estimated according to the experience of decision makers, so a higher weight is assigned to it. That is $\omega_{1}, \omega_{2}$ and $\omega_{3}$ are respectively equal to $1 / 6,2 / 3,1 / 6$, and $\alpha=0.5$. 
Based on this, the right side of Equation (14) can be used to replace the corresponding fuzzy variables in the Equation (9), so as to realize the defuzzification of model. That is:

$$
\sum_{j=1}^{m} x_{i j} \leq \omega_{1} \mathbf{a}_{i}+\omega_{2} \mathbf{b}_{i}+\omega_{3} \mathbf{c}_{i}
$$

\subsection{Algorithm Design}

Based on the defuzzification, the establishment of the above the multiple maintenance point emergency resource allocation multi-objective fuzzy constraint programming model is changed to certain multi-objective programming model. There are many methods for the solution of the multi-objective programming problem. This paper adopts the main target method, the hierarchical ordering method and the genetic algorithm convergence in the heuristic algorithm to solve this problem, the solving process is as follows:

Firstly, the main target method is used to deal with the model. The time satisfaction degree objective function for the Equation (5) is taken as the main objective function, and the demand satisfaction degree objective function for the Equation (6) is constrained and transformed by a certain way. Equation (6) can be equivalent to the following three conditions:

$$
\begin{gathered}
\sum_{j=1}^{m} h_{j}=\sum_{j=1}^{m} \sum_{i=1}^{n} x_{i j} \\
\left(\mathrm{R}_{i}-\mathrm{R}_{i}\right)\left(w_{\mathrm{i}}-w_{\mathrm{i}}\right) \geq 0, \mathrm{i}, \mathrm{i}^{\prime} \in I \\
\mathrm{R}_{i} \geq S I, \mathrm{i} \in I
\end{gathered}
$$

Equation (16) denotes that spare parts which play a role on the condition of the initial configuration of the emergency spare parts are in short supply of large-scale unexpectedness case. Equation (17) denotes limited emergency spare parts distribution criterion. Equation (18) ensures the distribution of the spare parts, and SL denotes the proportion of the minimum requirements of every maintenance station need to be supported. The values of SL are determined according to specific situation of the emergency.

Secondly, the method of hierarchical ordering is utilized to order according to degree of significance of the rest two objective function. In the initial stage of the emergency, quick response is able to minimize losses, and the response time become one of the key elements for dealing with emergencies. Therefore, the time satisfaction degree function is taken as the primary goal. The demand satisfaction degree is taken as the second goal. The emergency budget costs is taken as the third goal. The optimal solution set $S_{2}$ for the second objective 
function is got from the optimal solution set $S_{1}$ of the first objective function $\max \sum_{i=1}^{n} w_{i} \mathrm{~V}\left(T_{i}\right)$, and the solution of $S_{2}$ is taken as optimal solution.

Thirdly, genetic algorithm is a kind of solving technology to solve the optimal solution of the complex problem by using evolution and genetic, which has well features of high searching efficiency and solving the global optimal solution, and is widely used in the field of the current large-scale resource scheduling and allocation (Kim, Gen \& Yamazaki, 2003; Mendes, Gonçalves \& Resende, 2009; Su, Wu \& Yu, 2006). In this paper, based on the method of (Yan, Che, Li \& Yang, 2010), the method of introducing neighborhood search on the basis of crossover and mutation and chromosome filter search is proposed to improve the searching quality and efficiency of GA.

The main function of the algorithm is to filter chromosome not satisfy the constraint conditions before the operation of the choice of genetic algorithms.

Step 1. For generating initial population, the time cost of each chromosome of the population is compared with the maximum time cost. The service resource cost is compared with the largest service resources cost that customers are able to accept, and customer service satisfaction degree is compared with the minimum customer satisfaction degree. If there is a condition not satisfied, it will be removed from the population.

Step 2. If the exclusion of numbers of individuals is more than half of the population, it should be returned to Step 1. Otherwise, it should be returned to step 3 .

Step 3. The remaining population individuals are ranked according to the individual fitness, and high fitness individuals are chosen to reproduce.

In order to improve the convergence speed of the GA, after the operation of crossover and mutation, the individual of the best fitness neighborhood is chosen as individuals of offspring which is taken as a basis for neighborhood search. Population size is set to pop number.

Step 1. To make $i=0, j=0$.

Step 2. If $i>$ popnumber, it will turn to Step 9, or turn to Step 3.

Step 3. If the $j$ th gene-bit is unable to meet the last of the current chromosome, it will turn to Step 4, or turn to Step 7.

Step 4. Starting with the first a chromosome of population, the $j$ th genotypic value $x_{j}$ of it plus one.

Step 5. The fitness value of the ith chromosome is fitness $s_{i j}$.

Step 6. To make $j=j+1$, and it will turn to Step 3 . 
Step 7. If the fitness value of the transformed chromosomes is less than the fitness value of the previous transition chromosomes, it need to replace chromosomes.

Step 8. To make $i=i+1, j=0$, it will turn to Step 2 .

Step 9. The neighborhood search is completed.

\section{Simulation Example}

There are ten emergency maintenance stations and four spare parts warehouse centers in a large equipment company, and the fuzzy demand vectors of all emergency maintenance stations (expressed by A I) after the emergency events happen as shown in Table 1.

\begin{tabular}{|c|c|}
\hline $\begin{array}{c}\text { Emergency maintenance } \\
\text { station }\end{array}$ & Spare parts fuzzy demand \\
\hline A & $(10,13,15)$ \\
\hline B & $(15,17,20)$ \\
\hline C & $(16,18,20)$ \\
\hline D & $(14,18,21)$ \\
\hline E & $(11,13,16)$ \\
\hline F & $(13,15,18)$ \\
\hline G & $(15,17,19)$ \\
\hline H & $(16,19,21)$ \\
\hline I & $(14,17,20)$ \\
\hline J & $(14,15,16)$ \\
\hline
\end{tabular}

Table 1 . The fuzzy demand of spare parts

When the emergency happens, according to the statistics of the existing emergency resource and demand, the weight vector after sorting the determined support priority by using the above information entropy theory is:

$$
w_{i}=\left(w_{1}, w_{2}, w_{3}, w_{4}, w_{5}, w_{6}, w_{7}, w_{8}, w_{9}, w_{10}\right)=(0.1,0.1,0.05,0.15,0.15,0.05,0.2,0.05,0.05,0.1)
$$

The existing storage for spare parts storage center are:

$$
h_{j}=\left(h_{1}, h_{2}, h_{3}, h_{4}\right)=(38,40,32,47)
$$


Based on (Kahneman \& Tversky, 1984), to take $S L=0.3, \alpha=0.88, \beta=0.88, \lambda=2.25$, $c^{*}=5000$. Time distance and transportation costs from each storage center to the maintenance station are presented in Table 2.

\begin{tabular}{|c|c|c|c|c|c|c|c|c|c|c|}
\hline \multirow{2}{*}{$\begin{array}{l}\text { Warehouse } \\
\text { center }\end{array}$} & \multicolumn{10}{|c|}{ Maintenance station } \\
\hline & 1 & 2 & 2 & 4 & 5 & 6 & 7 & 8 & 9 & 10 \\
\hline 1 & $1 / 5$ & $2 / 5$ & $5 / 3$ & $3 / 1$ & $4 / 2$ & $5 / 2$ & $3 / 5$ & $1 / 2$ & $3 / 6$ & $2 / 9$ \\
\hline 2 & $2 / 3$ & $2 / 6$ & $4 / 7$ & $4 / 5$ & $3 / 6$ & $5 / 4$ & $1 / 2$ & $2 / 9$ & $3 / 4$ & $5 / 7$ \\
\hline 3 & $4 / 6$ & $2 / 4$ & $3 / 6$ & $3 / 6$ & $1 / 4$ & $3 / 5$ & $2 / 6$ & $2 / 5$ & $4 / 6$ & $4 / 2$ \\
\hline 4 & $7 / 2$ & $3 / 8$ & $2 / 5$ & $3 / 9$ & $3 / 8$ & $5 / 7$ & $3 / 3$ & $3 / 9$ & $4 / 7$ & $5 / 2$ \\
\hline
\end{tabular}

Table 2. The distribution table of time and transportation cost (t/c)

The above data is taken to the multiple maintenance point emergency resource allocation model built up in this paper, using MATLAB2007 language programming, and the optimized solution set got by genetic algorithm toolbox is:

$$
x_{i j}=\left\{\begin{array}{cccccccccc}
0 & 0 & 11 & 0 & 0 & 11 & 0 & 10 & 6 & 0 \\
4 & 9 & 0 & 0 & 9 & 0 & 8 & 0 & 0 & 10 \\
0 & 9 & 0 & 10 & 5 & 5 & 0 & 0 & 0 & 3 \\
9 & 0 & 7 & 7 & 0 & 0 & 6 & 9 & 9 & 0
\end{array}\right\}
$$

The values of the objective function time satisfaction degree and demand satisfaction degree were obtained by calculation, which are 0.92 and 0.85 , respectively, and the emergency cost is 4785. The global optimal solutions of emergency spare parts allocation is as shown in Table 3.

\begin{tabular}{|c|c|c|c|c|c|c|c|c|c|c|}
\hline \multirow{2}{*}{$\begin{array}{l}\text { Warehouse } \\
\text { center }\end{array}$} & \multicolumn{10}{|c|}{ Maintenance station } \\
\hline & 1 & 2 & 2 & 4 & 5 & 6 & 7 & 8 & 9 & 10 \\
\hline 1 & - & - & 11 & - & - & 11 & - & 10 & 6 & - \\
\hline 2 & 4 & 9 & - & - & 9 & - & 8 & - & - & 10 \\
\hline 3 & - & 9 & - & 10 & 5 & 5 & - & - & - & 3 \\
\hline 4 & 9 & - & 7 & 7 & - & - & 6 & 9 & 9 & - \\
\hline
\end{tabular}

Table 3. The optimal solution for allocation of emergency spare parts 
The calculation result indicates that the multiple maintenance point emergency spare parts support model was built up in this paper under the condition of fuzzy demand, which can effectively solve the emergency resource limited multiple maintenance point resource allocation scheme, and meet time and demand satisfaction degree of customer maximum. Comparing traditional GA with the algorithm proposed in this paper from two aspects of solving speed and the value of the objective function, it can be seen that the solution speed of the proposed algorithm is fast while the number of iterations change from 100 to 500, the result was shown in Figure 3. In terms of solving the optimal function values, the number of iterations of each algorithm is set by 100 to 500, and it can be seen from the Figure 4 that the proposed algorithm is superior to GA.

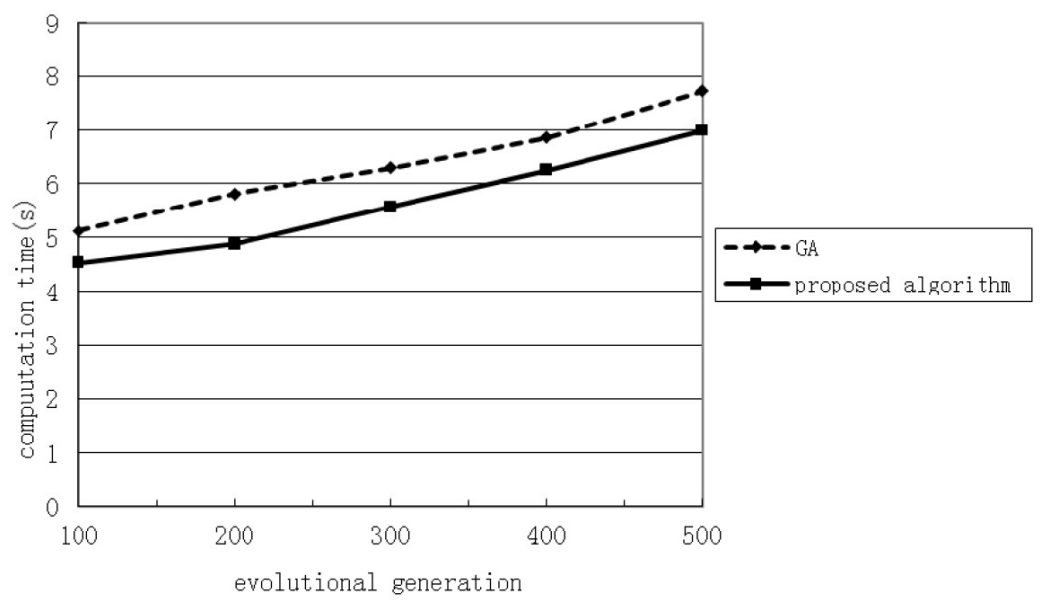

Figure 3. Efficiency comparisons among various algorithms

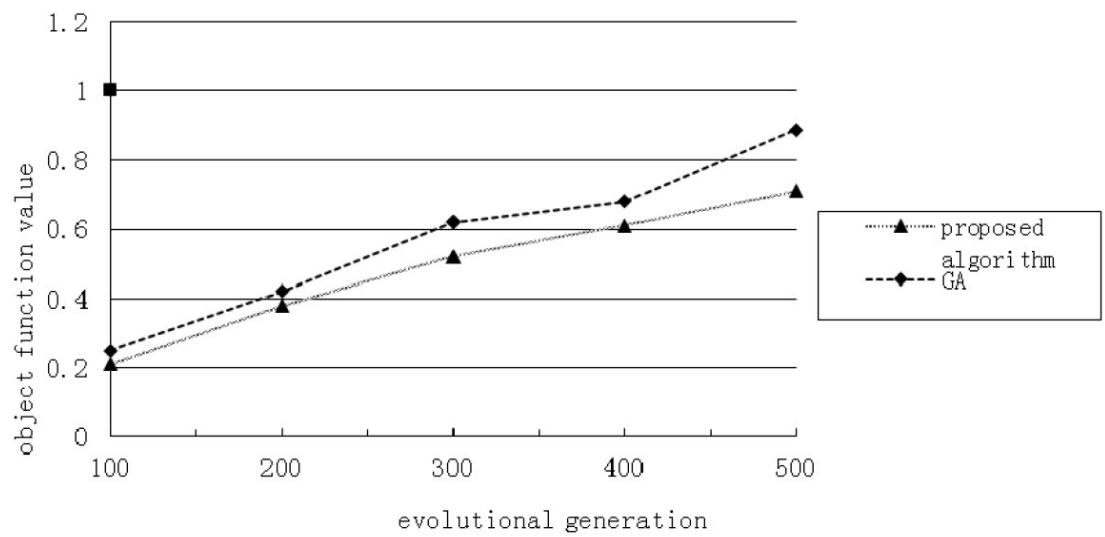

Figure 4. Comparisons of target values among various algorithms 


\section{Conclusion}

When the emergency event happens, it is very important to response rapidly for the work of maintenance spare parts for large equipment. The demand of spare parts is vague, and the existing resources such as maintenance personnel, spare parts storage and transportation tools are limited, so we need to conduct support prioritization according to the degree of urgency and the importance of maintenance station. In order to overcome the volatility and inaccuracy of information data. The theory of information entropy was used to conduct the weight sorting for emergency maintenance station support priority. The traditional method usually puts particular emphasis on the emergency decision making problems of the shortest response time, which is based on the method of quantified emergency response time and demand satisfaction degree, and the multiple maintenance points emergencies resource allocation model under the condition of fuzzy demand of considering time satisfaction degree and demand satisfaction degree, which combines the method of multi-objective programming with genetic algorithm The validity of the model is proved by a real instance, and the solution of emergency spare parts support is given under the condition of emergency events.

\section{Acknowledgments}

The work reported in this paper is supported by National Nature Science Foundation of China under grant NO. 71231001.

\section{References}

Dai, G.X., \& Da, Q.I. (2000). The Study of Combinatorial Scheduling Problem in Emergency Systems. Systems Engineering-theory \& Practice, 20(9), 52-55.

Du, M., \& Yi, H. (2013). Research on multi-objective emergency logistics vehicle routing problem under constraint conditions. Journal of Industrial Engineering and Management, 6(1), 258-266. http://dx.doi.org/10.3926/jiem.670

Fiedrich, F., Gehbauer, F., \& Rickers, U. (2000). Optimized resource allocation for emergency response after earthquake disasters. Safety Science, 35(1), 41-57.

http://dx.doi.org/10.1016/S0925-7535(00)00021-7

Kahneman, D., \& Tversky, A. (1984). Choices, values, and frames. American psychologist, 39(4), 341-349. http://dx.doi.org/10.1037/0003-066X.39.4.341

Kim, K.W., Gen, M., \& Yamazaki, G. (2003). Hybrid genetic algorithm with fuzzy logic for resource-constrained project scheduling. Applied Soft Computing, 2(3), 174-188. http://dx.doi.org/10.1016/S1568-4946(02)00065-0 
Li, L., \& Lai, K.K. (2000). A fuzzy approach to the multiobjective transportation problem. Computers \& Operations Research, 27(1), 43-57. http://dx.doi.org/10.1016/S0305-0548(99)00007-6

Liang, T.F. (2006). Distribution planning decisions using interactive fuzzy multi-objective linear programming. Fuzzy Sets and Systems, 157(10), 1303-1316.

http://dx.doi.org/10.1016/j.fss.2006.01.014

Liu, C.L., He, J.M., \& Shi, J.J. (2001). The Study on Optimal Model for a Kind of Emergency Material Dispatch Problem. Chinese Journal of Management Science, 9(3), 29-36.

Mendes, J.J.D.M., Gonçalves, J.F., \& Resende, M.G. (2009). A random key based genetic algorithm for the resource constrained project scheduling problem. Computers \& Operations Research, 36(1), 92-109. http://dx.doi.org/10.1016/j.cor.2007.07.001

Özdamar, L., Ekinci, E., \& Küçükyazici, B. (2004). Emergency logistics planning in natural disasters. Annals of operations research, 129(1-4), 217-245.

http://dx.doi.org/10.1023/B:ANOR.0000030690.27939.39

Shen, J.B. (2007). An emergency logistics distribution approach for quick response to urgent relief demand in disasters. Transportation Research Part E: Logistics and Transportation Review, 43(6), 687-709. http://dx.doi.org/10.1016/j.tre.2006.04.004

Su, P., Wu, N.Q., \& Yu, Z.Q. (2006). Application of Improved Genetic Algorithm in Partner Selection and Optimization for a Virtual Enterprise. Systems Engineering-theory \& Practice, 26(12), 404-410.

Tzeng, G.H., Cheng, H.J., \& Huang, T.D. (2007). Multi-objective optimal planning for designing relief delivery systems. Transportation Research Part E: Logistics and Transportation Review, 43(6), 673-686. http://dx.doi.org/10.1016/j.tre.2006.10.012

Wang, X.P., Ma, C., \& Ruan, J.H. (2013). Model and algorithm of relief materials dynamic scheduling without sufficient vehicle quantity. Systems Engineering-theory \& Practice, 33(6), 1492-1500.

Yan, P.Y., Che, A.D., Li, P. \& Yang, N.D. (2010). Improved genetic algorithm for robotic cell schedul ing problem with flexible processing times. Computer Integrated Manufacturing System, 2, 404-410.

Journal of Industrial Engineering and Management, 2015 (www.jiem.org)

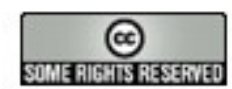

Article's contents are provided on a Attribution-Non Commercial 3.0 Creative commons license. Readers are allowed to copy, distribute and communicate article's contents, provided the author's and Journal of Industrial Engineering and Management's names are included. It must not be used for commercial purposes. To see the complete license contents, please visit http://creativecommons.org/licenses/by-nc/3.0/. 